\title{
Dynamics of two particles on a vertically driven plate
}

\author{
Kai Yang ${ }^{1}$, Alex Sabey ${ }^{1}$, and Jeffrey Olafsen ${ }^{1 *}$ \\ ${ }^{1}$ Department of Physics, Baylor University, One Bear Place \#97356, Waco, TX 76798, USA
}

\begin{abstract}
We experimentally investigate the dynamics of two particles driven vertically and free to collide in a nearly one-dimensional channel. Both spherical particles are Delrin with a diameter of $d=5.0 \mathrm{~mm}$. We obtained data for driving frequencies, $f$, ranging from 26.06 to $29.52 \mathrm{~Hz}$ and acceleration magnitudes, $\Gamma$, from 1.79 to $2.42 \mathrm{~g}$. High speed digital imaging is used to extract the positions of the two particles from experiment, with digital imaging analysis used to extract velocities and accelerations from sequential images. The total energy and the velocity of the particles before and after collisions are studied in both the lab frame and centre of mass frame. Most of the experimental results suggest a linear dependence for the relative velocity after collision on the relative velocity before collision with the coefficient of restitution approximately constant at 0.8 . However, some data in the low impact velocity regime indicates the possibility of an occasional energy transfer between the rotational and translational degrees of freedom.
\end{abstract}

\section{Introduction}

A bouncing ball driven vertically on a vibrating platform under gravity has received a lot of attentions since the pioneering work of Luck and Mehta [1]. Despite the simplicity of this dynamical system, a rich variety of behaviours may occur including periodic motion, period-doubling cascade and chaos [2]. Understanding these behaviours requires a precise knowledge of the inelasticity characterized by the coefficient of restitution, e [3], which is defined as the ratio between the rebound velocity and the impact velocity:

$$
e=v_{\text {out }} / v_{\text {in }}
$$

where $v_{\text {out }}$ is the rebound velocity and $v_{\text {in }}$ is the impact velocity. Recently, many investigations have shown that the coefficient of restitution is not a simple material constant but depends on the material properties and the impact velocities [4]. Typically, the coefficient of restitution is defined in the range of $0 \leq e \leq 1$, but in some special cases like oblique collisions [5] and nanoparticle collisions [6], the coefficient of restitution may exceed unity. Such behavior can be described as the super-elastic collisions. Depending on the impact geometry, the coefficient of restitution can also be observed to have negative values [7]. Numerous investigations have been developed to experimentally and numerically study single particle dynamics including using aspherical and grain-filled particles [810], a plate with concave surface [11], wet particles and dry spheres colliding with liquid layers [12, 13]. However, studies on two particle collision dynamics are still scarce and would provide significant insight into the detailed mechanisms of energy dissipation that are important in granular systems of larger particle number.
In this paper, we use two Delrin particles driven on a horizontal plate free to collide vertically in a nearly one-dimensional channel to study the particle-particle collisions. Data are obtained for driving frequencies, $f$, ranging from 26.06 to $29.52 \mathrm{~Hz}$ and the acceleration magnitudes, $\Gamma$, from 1.79 to $2.42 \mathrm{~g}$. The behaviour of the collisions is examined in both the lab frame as well as the centre of mass frame for the two identical colliding particles.

\section{Experiment}

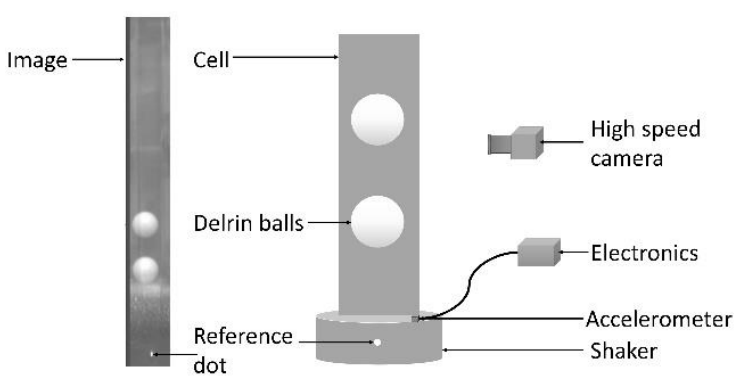

Fig. 1. The experimental setup. Two Delrin particles are placed in a nearly one-dimensional channel and driven vertically by a shaker. The positions and velocities of the two particles and the reference dot attached to the cell platform are extracted by analysis of images from the high-speed camera.

\subsection{Experimental setup}

Our experimental setup, see Fig. 1, includes a shaker, electronics, and an enclosed nearly one-dimensional cell

\footnotetext{
* Corresponding author: Jeffrey_Olafsen@baylor.edu

A video is available at https://doi.org/10.48448/2xmj-0c44
} 
with two identical Delrin particles confined to move in the vertical direction. The dimensions of the channel to the particle size do not allow the particles to move past one another. The diameter of each of the Delrin particles is $5.0 \mathrm{~mm}$. A bright dot on the shaker is used as the reference dot. The shaker is driven vertically by a sin wave from the electronics with the displacement $y(t)=$ $A \sin (\omega t)$, where $\omega=2 \pi f, A$ and $f$ are the amplitude and frequency of the vibration respectively. The acceleration is monitored by an accelerometer mounted on the shaker platform and can be normalized to the gravitational acceleration $g=9.8 \mathrm{~m} / \mathrm{s}^{2}$, denoted by $\Gamma=A \omega^{2} / g$. High-speed digital imaging with a rate of 353.5 frames $/ \mathrm{s}$ is used to capture the positions both the two Delrin particles and the reference dot. Image analysis is then used in sequential images to extract velocity and acceleration measurements.

The experiment is conducted by shaking the two particles at a fixed frequency $f$ and amplitude $\Gamma$. Data that will be shown in this paper are for the parameters $f_{1}=29.52 \mathrm{~Hz}, \Gamma_{1}=2.42 \mathrm{~g} ; f_{2}=26.17 \mathrm{~Hz}, \Gamma_{2}=$ $2.385 \mathrm{~g} ; f_{3}=26.06 \mathrm{~Hz}, \Gamma_{3}=1.79 \mathrm{~g}$, respectively.

\subsection{Experimental results}

The vertical positions of the two particles and the reference dot are extracted from the images and plotted as a function of time as shown in Fig. 2. Most of the collisions are identified and highlighted with diamond symbols. The position of the reference dot is fitted to a sinusoidal function, allowing measurements of the driving frequency and amplitude to be compared with the image data. The driving frequency and amplitude measured by the accelerometer in the data shown case are $f_{2}=26.17 \pm 0.05 \mathrm{~Hz}$ and $\Gamma_{2}=2.385 \pm 0.010 \mathrm{~g}$, comparing well to $f_{2}{ }^{*}=26.09 \pm 0.30 \mathrm{~Hz}$ and $\Gamma_{2}{ }^{*}=$ $2.19 \pm 0.23 g$ obtained by the image data.

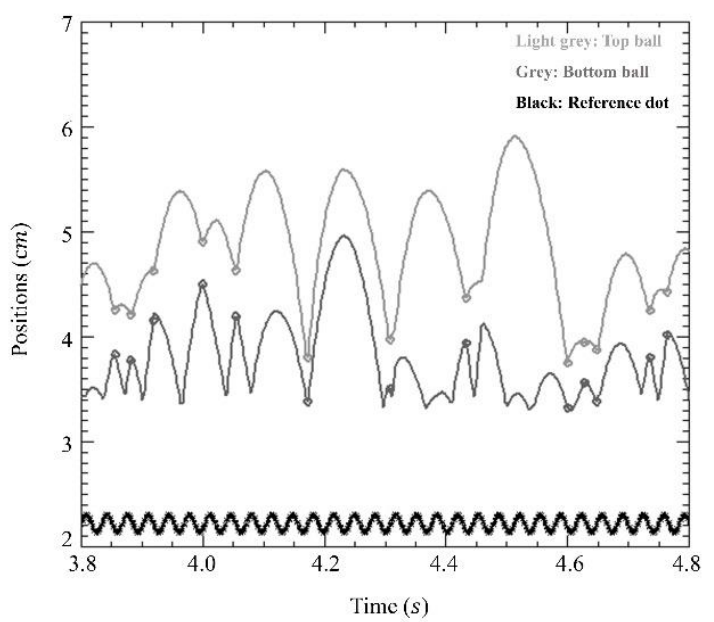

Fig. 2. Ball and plate positions plotted versus time.

Fig. 3 shows the relative velocity before and after collision in the lab frame. Most of data $(\sim 70 \%)$ are below the diagonal line for all 3 cases with the coefficient of restitution smaller than 1 , while the rest data scattered above the diagonal line shows the coefficient of restitution exceeds unity (the so called super-elastic collisions). We speculate these collisions are due to the exchange of energy between the rotational and translational degree of freedom. Since only the translational energy is used to calculate the coefficient of restitution, the relative velocity after collision may be greater than the relative velocity before collision due to the gaining of translational energy at the expense of the rotational energy. This process should also work in reverse in freely colliding particles, implying a loss of translational energy to the rotational mode in certain circumstances. The scatter of the data suggests a strong dependence of the coefficient of restitution on the impact velocity. It is worth noticing that in Fig. 3 (c), there is an increasing density of data around the diagonal line as the impact velocity decreases to zero. At such small speeds these energy transfers may be more significant.
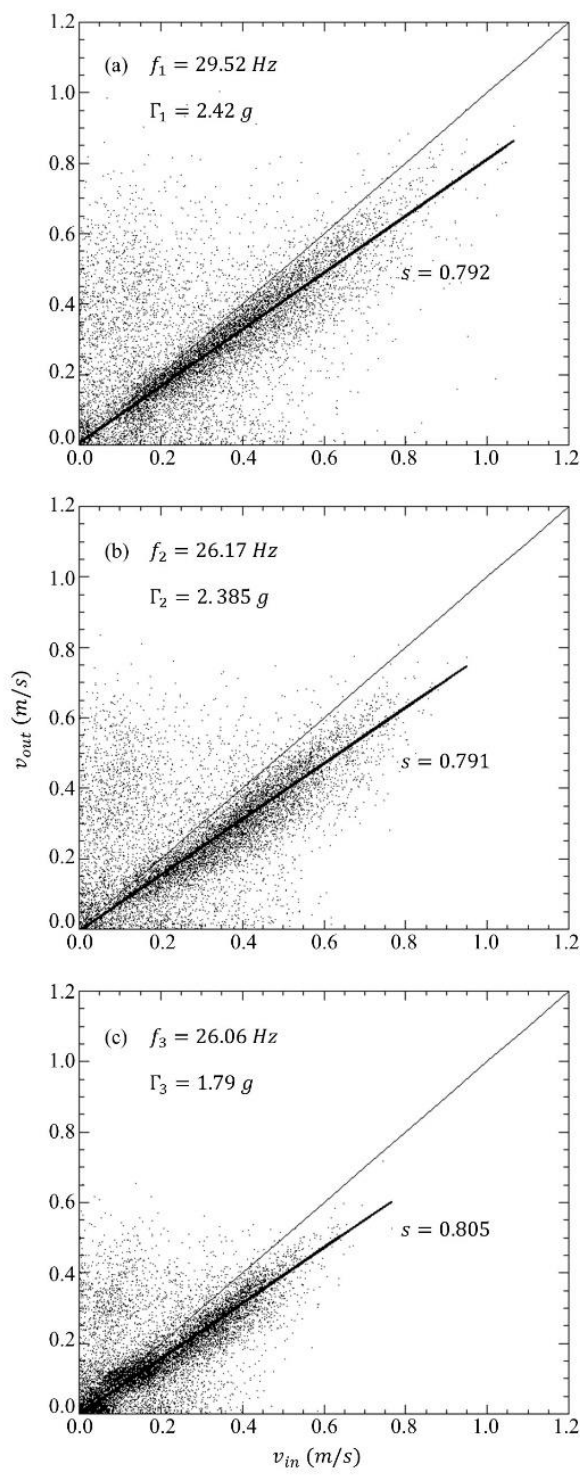

Fig. 3. Plot of relative velocity after collision $v_{\text {out }}$ against the relative velocity before collision $v_{i n}$ in the lab frame for all data. Parameters are as follows: (a) $f_{1}=29.52 \mathrm{~Hz}, \Gamma_{1}=$ $2.42 \mathrm{~g}$; (b) $f_{2}=26.17 \mathrm{~Hz}, \Gamma_{2}=2.385 \mathrm{~g}$; (c) $f_{3}=26.06 \mathrm{~Hz}$, $\Gamma_{3}=1.79 \mathrm{~g}$. 

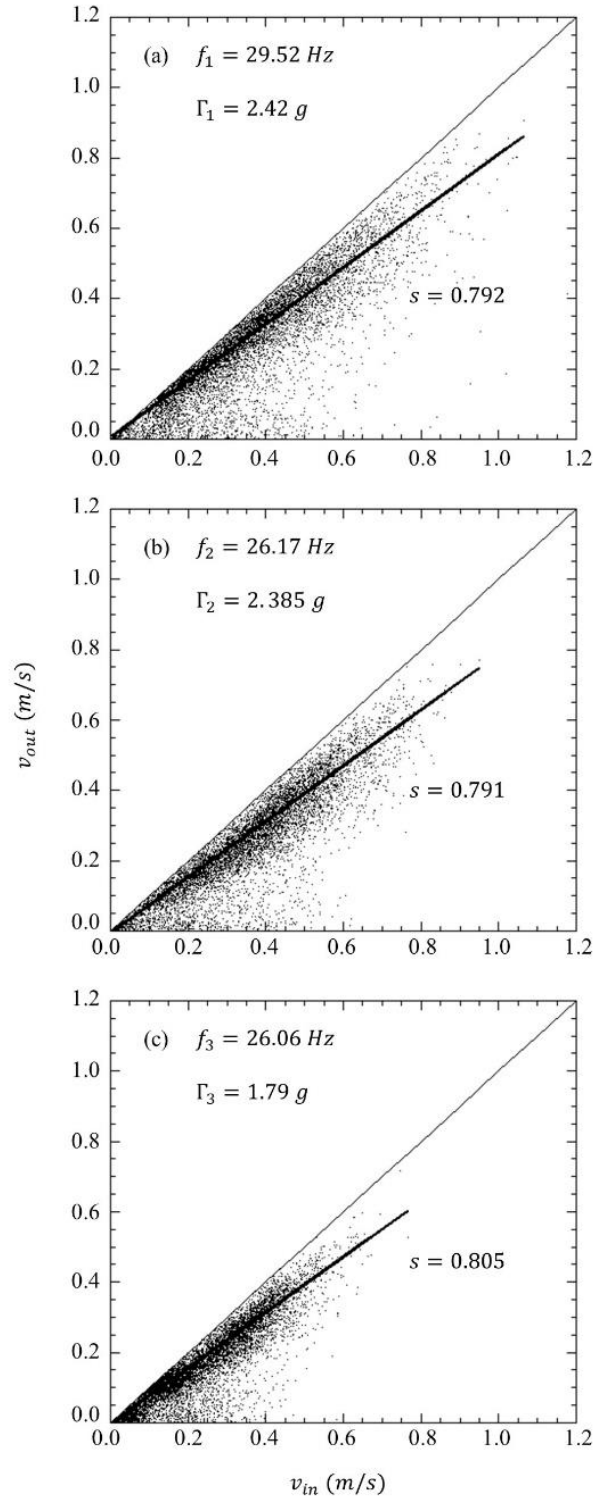

Fig. 4. Plot of relative velocity after collision $v_{\text {out }}$ against the relative velocity before collision $v_{i n}$ in the lab frame for the data with coefficient of restitution less than or equal to 1 . Parameters are as follows: (a) $f_{1}=29.52 \mathrm{~Hz}, \Gamma_{1}=2.42 \mathrm{~g}$; (b) $f_{2}=26.17 \mathrm{~Hz}, \Gamma_{2}=2.385 \mathrm{~g}$; (c) $f_{3}=26.06 \mathrm{~Hz}, \Gamma_{3}=$ $1.79 \mathrm{~g}$.

Fig. 4 shows the same data for the relative velocity after collisions versus the relative velocity before collisions in the lab frame with the super-elastic collisions excluded. This represents about $70 \%$ of the data which cluster around a straight line with a slope of nearly $s=0.8$ in all three cases. This suggests a mostly linear dependence for the relative velocity after collision on the relative velocity before collision and the coefficient of restitution is around 0.8 in the experiment. Comparing the results of Fig. 4 (a) and Fig. 4 (b), it seems to suggest that the coefficient of restitution is not sensitive to the driving frequency. Moreover, a comparison of the results from Fig. 4 (b) and Fig. 4 (c) seems to suggest only the slightest increase of the slope with a significant decrease of the driving amplitude.
While omitting data above the diagonal line excludes super-elastic collisions, it does not preclude the inclusion of data where kinetic energy could be lost to the rotational mode, resulting in a decreased postcollision velocity. A more careful analysis is clearly warranted to more closely examine the different types of collisions in this experiment. Since the rotational degree of freedom was not tracked in this experiment, we can instead re-examine the same data not in terms of the vertical velocity alone, but in terms of the total energy of the system.

The mechanical energy of the two particles consists of both the kinetic energy and potential energy, as well. Since the interactions in this experiment are free collisions of two identical particles, a centre of mass frame analysis is useful. Assuming both of the Delrin balls have the identical mass, $m$, before the collision, the bottom ball has a height of $y_{1}$ and the top ball with the height of $y_{2}$ with bottom edge of the image regarded as the zero potential position. The mechanical energy of the two balls before collision in the centre of mass frame can then be calculated as

$$
E_{\text {in }}=\frac{1}{2} \mu v_{i n}^{2}+m g\left(y_{1}+y_{2}\right)
$$

where $\mu=\frac{1}{2} m$ is the reduced mass, and $v_{i n}$ is the relative velocity of the two balls before collision. Similarly, assuming the heights of the bottom ball and top ball are $y_{1}^{\prime}$ and $y_{2}^{\prime}$ respectively after collision, the mechanical energy after the collision in the centre of mass frame is

$$
E_{\text {out }}=\frac{1}{2} \mu v_{\text {out }}^{2}+m g\left(y_{1}^{\prime}+y_{2}^{\prime}\right)
$$

where $v_{\text {out }}$ is the relative velocity after collision.

By normalizing the total energy to the mass $m$, then Eq. (2) and Eq. (3) would take the form

$$
\begin{gathered}
\frac{E_{\text {in }}}{m}=\frac{1}{4} v_{\text {in }}^{2}+g\left(y_{1}+y_{2}\right) \\
\frac{E_{\text {out }}}{m}=\frac{1}{4} v_{\text {out }}^{2}+g\left(y_{1}^{\prime}+y_{2}^{\prime}\right)
\end{gathered}
$$

This normalization is chosen as it reflects the analysis extracted from the image data.

Fig. 5 shows the normalized mechanical energy after collision $E_{\text {out }} / \mathrm{m}$ as a function of the mechanical energy before collision $E_{i n} / m$, for $f_{1}=29.52 \mathrm{~Hz}, \Gamma_{1}=$ $2.42 \mathrm{~g} ; f_{2}=26.17 \mathrm{~Hz}, \Gamma_{2}=2.385 \mathrm{~g} ;$ and $f_{3}=$ $26.06 \mathrm{~Hz}, \Gamma_{3}=1.79 \mathrm{~g}$. In all three cases, most of the data cluster slightly below the diagonal line, which indicate a slight mechanical energy loss during the collisions to plastic deformation and heat. It is observed that fluctuations exist for the data along the diagonal line in all three cases which is probably due to the microscopic surface roughness of the spheres and the air drag. The data scattered on each side of the diagonal line also suggest the transferring of energy between the rotational and translational degree of freedom is mostly, but not completely, symmetric. The more significant scatter of data occurs in the low impact energy regime, where any transfer of energy from the rotational degree of freedom would be more significant relative to the small translational energy. This scatter is less prevalent at higher energies in a similar manner when the rotational kinetic energy is then smaller than the translational energy of the particles. 

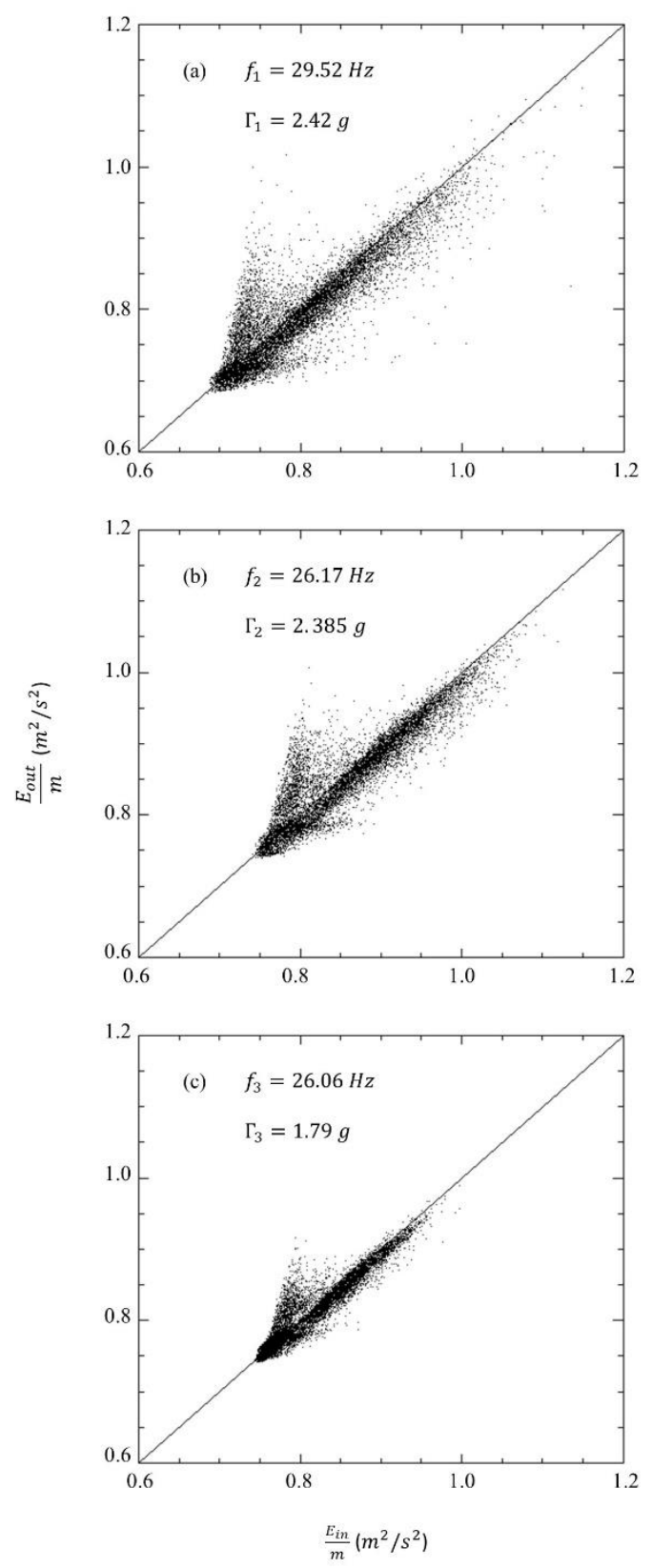

Fig. 5. Normalized mechanical energy after collision $\frac{E_{\text {out }}}{m}$ against the normalized mechanical energy before collisions $\frac{E_{i n}}{m}$ in the center of mass frame; Parameters are as follows: (a) $f_{1}=29.52 \mathrm{~Hz}, \Gamma_{1}=2.42 \mathrm{~g}$; (b) $f_{2}=26.17 \mathrm{~Hz}, \Gamma_{2}=$ $2.385 \mathrm{~g}$; (c) $f_{3}=26.06 \mathrm{~Hz}, \Gamma_{3}=1.79 \mathrm{~g}$.

\section{Conclusions}

We studied the dynamics of two Delrin particles driven vertically in a nearly one-dimensional channel. Data are obtained for frequencies, $f$, ranging from 26.06 to $29.52 \mathrm{~Hz}$ and the acceleration magnitudes, $\Gamma$, from 1.79 to $2.42 \mathrm{~g}$. In the lab frame for the relative velocities before and after collisions, most data indicate the coefficient of restitution is smaller than unity. Among the data with coefficient of restitution smaller than 1 , about $70 \%$ of the data cluster near the straight line with a slope $s=0.8$, which suggest a linear dependency of the relative post-collision velocity on the relative velocity before collisions. The rest of the data scattered on top of the diagonal line indicates the coefficient of restitution is greater than unity and we speculate it is due to the possible and occasional energy transfer from the rotational to the translational degree of freedom. A smaller amount of scatter below the diagonal in the centre of mass frame would also seem to indicate some collisions have more loss to a less frequent transfer of energy from the translational to the rotational mode. We also note that the significant scatter in the data occurs primarily where the impact energy is small. The fluctuation of the data might also be attributed to the microscopic surface roughness of the balls and air drag, both being more important at lower velocities. In the centre of mass frame for the energy before and after collision, most of the data cluster slightly below the diagonal line, which indicates a linear dependency of the energy after collision on the energy before collision and a slight energy loss for most collisions. The balance of the small amount of data scattered on each side of the diagonal line indicates possible occasional energy transfer between the rotational and translational degrees of freedom. The measurement of the rotation of the particles in a future experiment and a more thorough and strict analysis to detail the related energy exchange between the rotational and translational degrees of freedom are the next steps on this research effort.

\section{References}

[1] J.M. Luck, A. Mehta, Phys. Rev. E 48, 5 (1993)

[2] Z.H. Jiang, Z.J. Liang, D.W. Zhou, Y.J. Deng, Physica A 548, 123875 (2020)

[3] J.J. Barroso, M.V. Carneiro, E.E.N.Macau, Phys. Rev. E 79, 026206 (2009)

[4] T. Schwager, T. Poschel, Phys. Rev. E 78, 051304 (2008)

[5] M.Y. Louge, M.E. Adams, Phys. Rev. E 65, 021303 (2002)

[6] R. Murakami, H. Hayakawa, Phys. Rev. E 89, 012205 (2014)

[7] P. Muller, D. Krengel, T. Poschel, Phys. Rev. E 85, 012205 (2012)

[8] A. Gilelmo, N. Gunkelmann, T. Poschel, Phys. Rev. E 90, 052204 (2014)

[9] Y. Kubo, S. Inagaki, M. Ichikawa, K. Yoshikawa, Phys. Rev. E 91, 052905 (2015)

[10] F. Pacheco-Vazquez, F. Ludewig, S. Dorbolo, Phys. Rev. Lett 113, 118001 (2014)

[11] J.Y. Chastaing, G. Pillet, N. Taberlet, J.C. Geminard, Phys. Rev. E 91, 052918 (2015)

[12] F. Gollwitzer, I. Rohberg, C.A. Kruelle, K. Huang, Phys. Rev. E 86, 011303 (2012)

[13] J.L. Ma, D. Y. Liu, X.P. Chen, Phys. Rev. E 88, 033018 (2013) 